Article

\title{
Determination of composite properties in the presence of fiber deboning and matrix damage by using the method of cell
}

Mohammad Ghalandari ${ }^{{ }_{1}, 2}$, Hossein Toozandehjani ${ }^{3}$, Ibrahim mahariq ${ }^{4}$,Thabet Abeljawad ${ }^{5,6,7}$

${ }^{1 *}$ corresponding: Institute of Research and Development, Duy Tan University, Da Nang 550000, Viet Nam

${ }^{2}$ Faculty of Electrical-Electronic Engineering, Duy Tan University, Da Nang 550000, Viet Nam Mohammadghlandari@duytan.edu.vn

${ }^{3}$ Assistant Professor Faculties of Mechanical and Mechatronics Engineering h.toozandehjani@shahroodut.ac.ir

${ }^{4}$ College of Engineering and Technology, American University of the Middle East, Kuwait Ibrahim.Maharik@aum.edu.kw

${ }^{5}$ Department of Mathematics and General Sciences, Prince Sultan University, P. O. Box 66833, Riyadh 11586, Saudi Arabia.

${ }^{6}$ Department of Medical Research, China Medical University, Taichung 40402, Taiwan.

${ }^{7}$ Department of Computer Science and Information Engineering, Asia University, Taichung, Taiwan. tabdeljawad@psu.edu.sa

\begin{abstract}
In this study, the elastic properties of composite materials are investigated, considering the effects of separation of fiber-matrix joint boundary and matrix failure. In this method, by assuming periodic microstructure and using a linear approximation of the displacement field by applying continuity and equilibrium conditions, the composite fiber composite relation is determined. The effect of separation is assumed by introducing tangential and normal scalar parameters in the equations by assuming the displacement field jump at the common boundary. In order to express the effect of matrix micro-cracks, the fracture mechanics framework of continuous environments was used and the microcracks parallel to the fibers, perpendicular to the fibers and in the thickness direction with scalar parameters were expressed. At the end of the effect of these parameters the results are presented in graphs. The results show that the presence of defective joint at the joint boundary and the matrix microcomponents reduce the hardness of the composite and thus it's bearing load, which is more significant at the defective joint state.
\end{abstract}


Keywords: Composite, Micromechanics, Cell Method, Failure, Isolation

\section{Introduction}

The use of composite materials has increased for half a century due to their unique properties such as strength, hardness, lightness and low cost. Also, the combination of different materials to achieve new properties gives designers a wide variety of applications. The industrial and commercial applications of composites are very diverse, some of which include aerospace, automotive, sports, mining and infrastructure. In addition, fiber-reinforced polymer composites in electronic components (circuit boards), construction (floor beams), sofas and mannequins (seat springs), power industries (transformer compartments), oil industry (oil rig design) and suction tubes, the medical industry (implants, bone plates, and dentures), power transmission shafts, and oxygen tanks.

Determining the effective mechanical properties of composite materials using the properties of components (matrices and fibers) has been of interest to researchers. In general, various factors such as the properties and volume percentages of the constituents and the joint boundary properties affect the composite properties. In general, various factors such as the properties and volume percentages of the constituents and the joint boundary properties affect the composite properties. The most important failure modes of the micro scale are matrix micro-crack, fiber-matrix joint separation, and fiber breakage [1].

In recent years, various models have been used to study the behavior of composites. According to the Voigt and Reuss model, the stiffness and softness properties of the mixing law are calculated and obtain the upper and lower strength limits [2,3]. Eshelby in 1957 presented a general solution for inclusions in the matrix and using their presented model the shear value and shear modulus was calculated [4]. In 1973, Mori and Tanaka [5] proposed the average strain of several inclusions in the matrix of the strain concentration tensors and then the bulk and shear modulus of the composite by his investigations are calculated. In the self-consistent method, the properties of two-phase composites are obtained by dissolving an inclusion in the infinite matrix [6]. Another valid model to predict the elastic modulus of composites is the Halpin-Tsai model [7], originally developed for fiber-reinforced composites based on the Hermes-Hill self-consistent model that is simplified by semi-empirical parameters with an acceptable accuracy. In addition to the classical methods mentioned, numerical methods based on finite element, finite boundary and finite difference sources are used to predict the elastic properties and stress / macro 
strains in a variety of composites [8-11]. These methods suffer from high computational volume and are not suitable for larger-scale complex geometries, despite the advantages of local field determination. In 1989, Aboudi introduced the cell method for determining the behavior of composite materials, in which the composite is considered periodic and consists of duplicate single cells [12]. This method, while simply providing a closed solution, predicts the elastic properties and can influence the influence of the joint boundary and matrix failure mechanisms on the properties in its flexible formulation. This method is a good candidate for multi-dimensional analysis and practical and industrial applications.

In the present study, the elastic properties of the composite were calculated using the cell method and the effect of the joint defect and matrix failure was investigated. First, the micromechanical formulation of the cell method is presented, the tangential and normal boundary separation parameters of the joints involved in the relationships, and also by applying the fracture mechanics of continuous environments, the influence of the matrix breakdown density on the reduction of the composite stiffness in the stiffness relation is applied. At the end of the properties diagrams are plotted in terms of fiber volume and different values of breakdown parameters.

\section{Micromechanical Basis of the Cell Method}

The unit cell model in question consists of 4 sub-cells, as shown in Figure 1. In this figure the $h, l$ overall dimensions of the unit cell and $h_{1}, h_{2}, l_{1}, l_{2}$ are dimensions of the cells. Generally, in single cell methods it is assumed that for a composite with periodic microstructure, the calculated properties for a cell containing fiber and matrix are equivalent to the total composite properties.

(a)

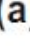

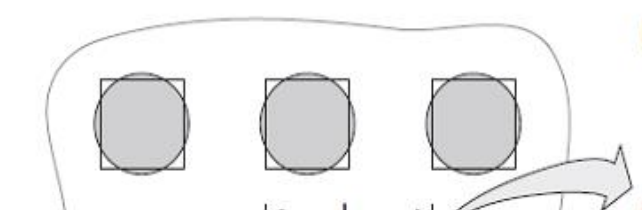

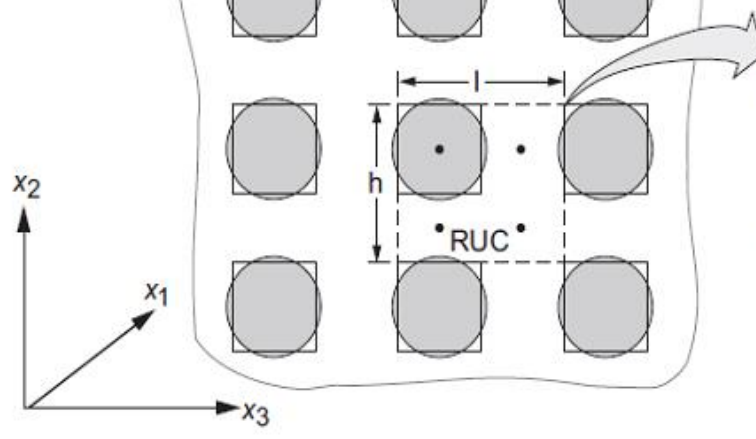

(b)

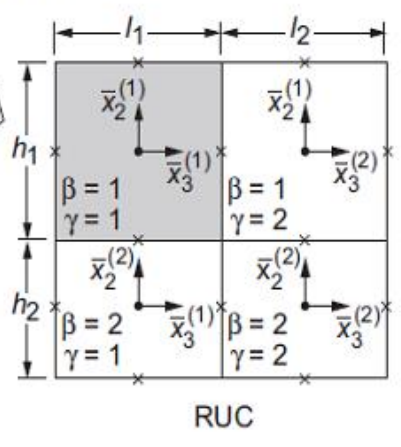

Figure 1: MOC model for continuous fiber composite. a) Periodic arrangement of fibers in the $x 1$ direction. $b$ ) Duplicate unit cells with 4 sub cells [12] 
The analysis of a duplicate single cell includes the stress vector coherence conditions and the displacement field at the common boundary between the sub cells as well as the neighboring unit cells and the static equilibrium conditions. One of the advantages of the cell method is the ability to calculate the properties of composites whose constituents exhibit nonlinear (corrosive or inelastic) behavior. The unit cell in this method consists of 4 square / rectangular sub-cells, one of which is fiber and the rest is matrix. So this method gives an approximation of the mechanical properties of the composite because the fiber is actually circular. However, in practical applications this approximation is in good agreement with the experimental data. The general and local coordinates and geometrical dimensions of the fiber and matrix sub-cells are shown in Fig. 1. In order to obtain the average behavior of the composite, a first-order theory is used to extend the displacement field in terms of the distance from the center of the substrates, $\bar{x}_{2}^{(\beta)}$ and $\bar{x}_{3}^{(\gamma)}$. Expansion field of displacement for each cell [12]:

$$
u_{i}^{(\beta \gamma)}=w_{i}^{(\beta \gamma)}(x)+\bar{x}_{2}^{(\beta)} \phi_{i}^{(\beta \gamma)}+\bar{x}_{3}^{(\gamma)} \psi_{i}^{(\beta \gamma)} \quad, \quad \mathrm{i}=1,2,3
$$

Where the $w_{i}^{(\beta \gamma)}(x)$ displacement components of the center of the cell $\phi_{i}^{(\beta \gamma)}$ and $\psi_{i}^{(\beta \gamma)}$ represent the linear dependence of the displacement on the local coordinates $\bar{x}_{2}^{(\beta)}$ and $\bar{x}_{3}^{(\gamma)}$ which are equivalent to the same shape functions in the finite element method. It should be noted that $\phi_{i}^{(\beta \gamma)}$ and $\psi_{i}^{(\beta \gamma)}$ do not depend on $x_{1}$.

The following equations are extracted by using the algebraic adaptation equation of displacement and applying the continuity condition at the joint boundary of the sub-cells.

$$
\begin{aligned}
& h_{1} \phi_{i}^{(1 \gamma)}+h_{2} \phi_{i}^{(2 \gamma)}=\left(h_{1}+h_{2}\right) \frac{\partial w_{i}^{(2 \gamma)}}{\partial x_{2}} \\
& l_{1} \psi_{i}^{(\beta 1)}+l_{2} \psi_{i}^{(\beta 2)}=\left(l_{1}+l_{2}\right) \frac{\partial w_{i}^{(\beta 2)}}{\partial x_{3}}
\end{aligned}
$$

Using the equations (1) and (2) and applying the condition $\frac{\partial w_{i}^{(\beta \gamma)}}{\partial x_{j}}=\frac{\partial w_{i}}{\partial x_{j}}$, we can find the relationship between the strain components and the micro variables: 


$$
\begin{aligned}
& \varepsilon_{11}^{(\beta \gamma)}=\frac{\partial}{\partial x_{1}} w_{1} \\
& \varepsilon_{22}^{(\beta \gamma)}=\phi_{2}^{(\beta \gamma)} \\
& \varepsilon_{33}^{(\beta \gamma)}=\psi_{3}^{(\beta \gamma)} \\
& 2 \varepsilon_{12}^{(\beta \gamma)}=\phi_{1}^{(\beta \gamma)}+\frac{\partial}{\partial x_{1}} w_{2} \\
& 2 \varepsilon_{13}^{(\beta \gamma)}=\psi_{1}^{(\beta \gamma)}+\frac{\partial}{\partial x_{1}} w_{3} \\
& 2 \varepsilon_{23}^{(\beta \gamma)}=\phi_{3}^{(\beta \gamma)}+\psi_{2}^{(\beta \gamma)}
\end{aligned}
$$

The mean strain volume is obtained from the following equation:

$$
\bar{\varepsilon}_{\mathrm{ij}}=\frac{1}{\mathrm{~V}} \sum_{\beta, \gamma=1}^{2} \mathrm{~h}_{\beta} 1_{\gamma} \varepsilon_{\mathrm{ij}}^{(\beta \gamma)}
$$

The cell volume is equal to $V=h l$. By writing the equation comprising each sub-cell, the stress-strain relation is determined. Assuming the transverse isotropic behavior of the matrix and the fiber, we can write the equation of the sub-cells as follows:

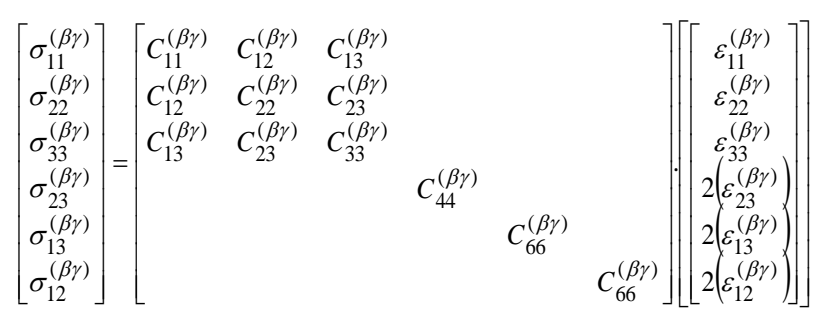

Where $C_{i j}^{(\beta \gamma)}$ is the stiffness matrix component. The volume average stress is obtained from the following relation:

$$
\bar{\sigma}_{i j}=\frac{1}{V} \sum_{\beta, \gamma=1}^{2} h_{\beta} l_{\gamma} \sigma_{\mathrm{ij}}^{(\beta \gamma)}
$$

After calculating the micro-variables in terms of geometrical parameters and general strains and using the volume average of the stress and strain tensors, one can write the general composite relationship of the unidirectional composite [12]:

$$
\bar{\sigma}=B\left(\bar{\varepsilon}-\alpha^{*} \Delta T\right)
$$

Where $\alpha^{*} \Delta T$ is the share of the thermal strain and $B$ the hardness matrix that is: 


$$
B=\left[\begin{array}{cccccc}
b_{11} & b_{12} & b_{13} & 0 & 0 & 0 \\
b_{12} & b_{22} & b_{23} & 0 & 0 & 0 \\
b_{13} & b_{23} & b_{33} & 0 & 0 & 0 \\
0 & 0 & 0 & b_{44} & 0 & 0 \\
0 & 0 & 0 & 0 & b_{55} & 0 \\
0 & 0 & 0 & 0 & 0 & b_{66}
\end{array}\right]
$$

The values of $b_{i j}$ are presented in detail in [12]. In this study, thermal deformation is ignored.

\subsection{Separation of common border}

The interface between fibers and matrices in composite materials is very important because the mechanical properties of fiber composites depend not only on the properties of the fibers but also on the degree of charge transfer to the fibers by the matrix and the stress transfer between the fibers and the matrix. It depends on the joint boundary and is determined by their degree of bonding and the joint boundary forces. Separation can occur during the manufacturing process or under load during service.

The effect of separating the common boundary between the fiber and the matrix within the cell method can be studied by assuming an intermediate phase layer between the fiber and the matrix. The thickness and properties of the intermediate phase can be determined by the quality of the joint boundary. However, the computation of the mid-phase properties is complicated, so for practical applications one can use the Jones [13] model, in which a small thickness flexible joint boundary is considered to model the fiber-matrix joint boundary separation. The scalar parameters $R_{t}$ and $R_{n}$ are expressed. In this method, the displacement field at the common boundary has a jump that is conditioned on the continuity of the common boundary. To express the separation phenomenon in the present micromechanical formulation, relation (2) is given as follows:

$$
\begin{aligned}
& h_{1} \phi_{i}^{(1 \gamma)}+h_{2} \phi_{i}^{(2 \gamma)}+2 R \bar{\sigma}_{2 i}^{(1 \gamma)}=h \frac{\partial w_{i}^{(2 \gamma)}}{\partial x_{2}} \\
& l_{1} \psi_{i}^{(\beta 1)}+l_{2} \psi_{i}^{(\beta 2)}+2 R \bar{\sigma}_{3 i}^{\beta 1}=l \frac{\partial w_{i}^{(\beta 2)}}{\partial x_{3}}
\end{aligned}
$$

Where $R=R_{n}$ or $R=R_{t}$ represents the normal and tangential separation parameters. By repeating the process of the previous section, these two parameters appear in the hard elements. In the $R_{t}=R_{n}=0$ state, the boundary of the joint boundary is complete and by increasing them first slip and then complete separation occur. According to the theory of $R_{t}=R_{n}=0$ values, denotes complete coupling, $R_{n}=0, R_{t} \rightarrow \infty$ 
denotes complete slip of common boundary and $R_{n}=\rightarrow \infty, R_{t} \rightarrow \infty$ denotes complete separation of common boundary.

\section{2- Micro-crack matrix}

Another common failure mechanism in composites is micro-crack matrix, which can increase the number of larger cracks and lead the composite to the final fracture stage. The presence of micro-cracks reduces the stiffness and composite strength, which can be explained by the failure mechanism of continuum mechanics. In this method Kachanov [14] introduced a failure variable that manifests itself as a decrease in the cross-section of the material. The effective cross-section of $\bar{A}$ is obtained by subtracting the levels occupied by the breakdowns of $A$. To do this, Kachanov [14] introduced a bulk element that is large enough on a macro scale to accommodate multiple failures, yet small enough to be considered a material point. The effective cross section is equal to:

$$
\bar{A}=(1-D) A
$$

Where $D=0$ is the material for non-failure state and $D=1$ for complete failure (failure). The cross sections of $\bar{A}, A$ are shown in Fig. 2.

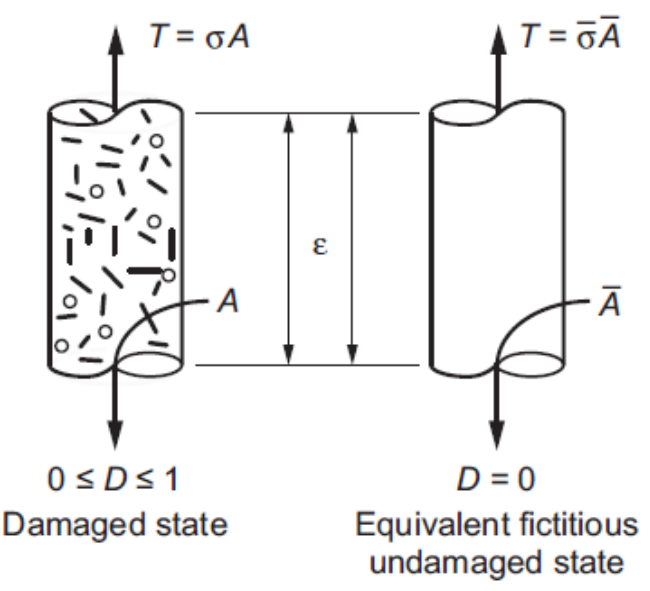

Figure 2 shows the breakdown in matter

According to the strain hypothesis, the equivalent of any shape change under single / multiaxial load on the material containing the failure can be expressed by the constitutive equation of 
the material without failure provided that the stress is replaced by the effective stress. The effective stress is [15]:

$$
\bar{\sigma}=\frac{\sigma}{(1-D)}
$$

Where $\mathrm{bb}$ is the effective stress in the failure state, the effect of the failure lies in the failure parameter. Therefore, in the presence of $0 \leq D \leq 1$ failure, the cross section is reduced by $(1-D)$ scale and the stress increases with this scale. The Hook's law for uniaxial stress is expressed as $\sigma=(1-D) E \varepsilon$. It should be noted that the generalization of the scalar parameter $D$ to the tensor form is also used to calculate the stiffness reduction in 3D and with different failure modes. In composite materials, micro-cracks can be perpendicular to the material axis, that is, parallel to the fibers, perpendicular to the fibers and in the direction of thickness. So assuming three parameters of $D_{1}, D_{2}, D_{3}$ failure, the deteriorated properties can be expressed as follows [16]:

\section{Results}

In this section, using the above-mentioned equations for a single-layer composite with glass / epoxy, boron / aluminum systems, the elastic properties and their changes are calculated in terms of the separation parameters and the matrix breakdown density. The material properties of fiber and matrix are listed in Table 1.

Table 1: Properties of fibers and matrices

\begin{tabular}{c|c|c|c}
\hline $\begin{array}{c}\text { Poisson } \\
\text { Ratio }\end{array}$ & $\begin{array}{c}\text { Young } \\
\text { Modulus } \\
(\mathrm{GPa})\end{array}$ & Matrix/Fiber & rows \\
\hline 0.22 & 73 & Glass & 1 \\
\hline 0.21 & 413 & Boron & 2 \\
\hline 0.34 & 3.42 & Epoxy & 3 \\
\hline 0.33 & 69 & Aluminum & 4 \\
\hline
\end{tabular}


In order to validate the method of the cells, the transverse and shear modulus modifications in terms of volume ratio were obtained and compared with the results in Reference [17], as shown in Figure 3. It is then plotted for glass / epoxy and boron / aluminum systems with a volumetric modulus of $30 \%$ and a transverse and axial shear modulus according to the tangent separation parameter of $\log \left(1+R_{t} G_{m} / h\right)$ for different volumetric ratios. As shown in Figures 4 and 5 , the increase in tangential separation reduces the properties, which is more significant for the $G_{A}$ axial shear modulus as expected, because increasing $R_{t}$ means that the boundary is slippery, so the fibers under load the $\gamma_{12}, \gamma_{13}$ shear cannot completely transfer the load from the matrix to the fibers. It can also be concluded that the effect of the incomplete joint boundary on the properties is independent of the fiber and matrix systems and the higher hardness constituents behave similarly.

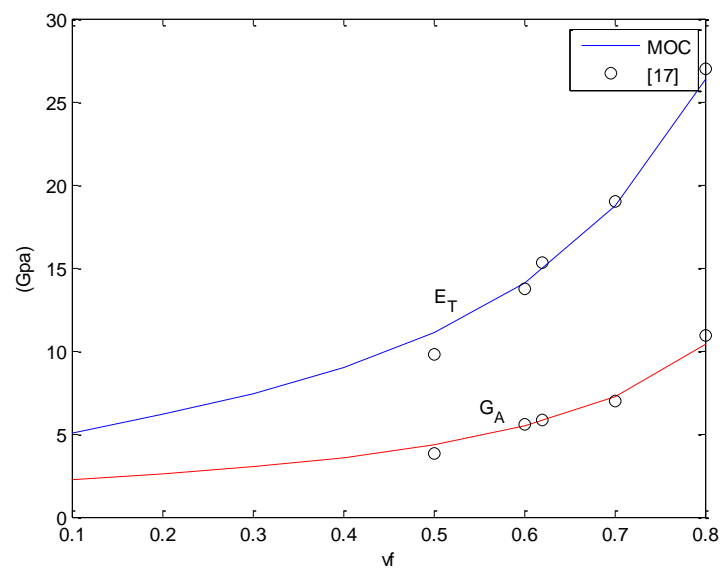

Figure 3. Comparison of changes of transverse modulus and axial shear modulus in terms of volume ratio with results [17]. 


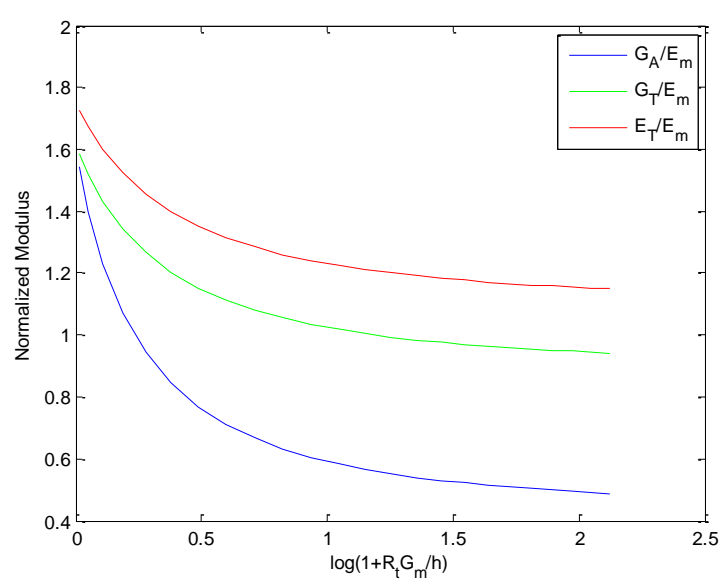

Figure 4. Changes of transverse modulus, transverse shear modulus, and axis of composite glass / epoxy with increasing tangential separation parameter

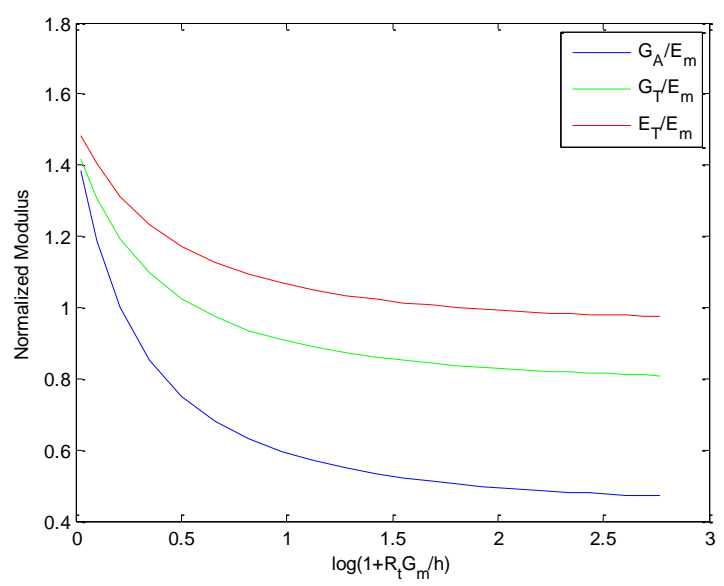

Figure 5. Changes of transverse modulus, transverse shear modulus, and axis of boron / aluminum composite with increasing tangential separation parameter

$R_{t}=0$ Changes of properties in terms of the $\log \left(1+R_{t} G_{m} / h\right)$ normal separation parameter for the epoxy / glass system are shown in Fig. 6. As can be seen, the normal separation did not affect $G_{A}$ and decreased the $G_{T}$ and $E_{T}$ values. This decrease in the composite transverse modulus is significant because by applying a transverse load to the composite and separating perpendicular to the joint boundary, the load transfer capacity from the matrix to the fiber decreases. 


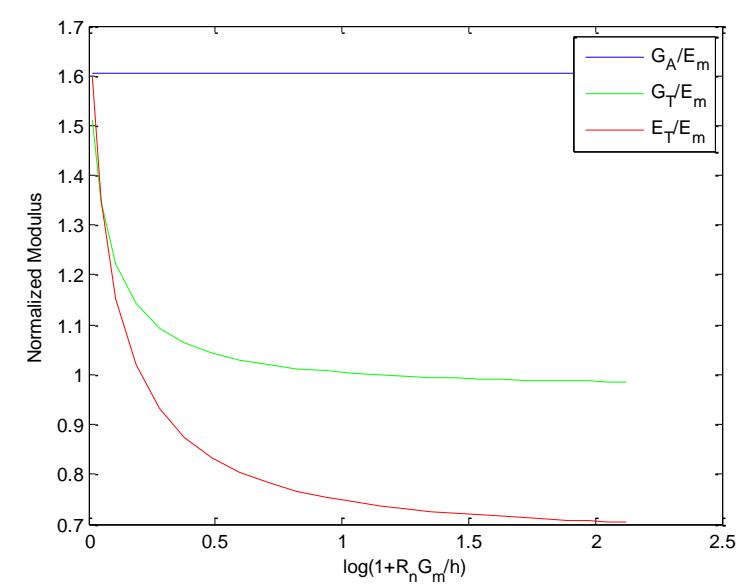

Figure 6. Changes of transverse modulus, transverse shear modulus, and axis of composite glass / epoxy with increasing normal separation parameter

If both separation parameters are changed, their effect on the properties can be studied simultaneously. Fig. 7 shows the decrease in transverse modulus relative to the simultaneous increase of $R_{t}$ and $R_{n}$. Given the shape effect of the $R_{n}$ in reducing the transverse modulus greater than $R_{n}$.

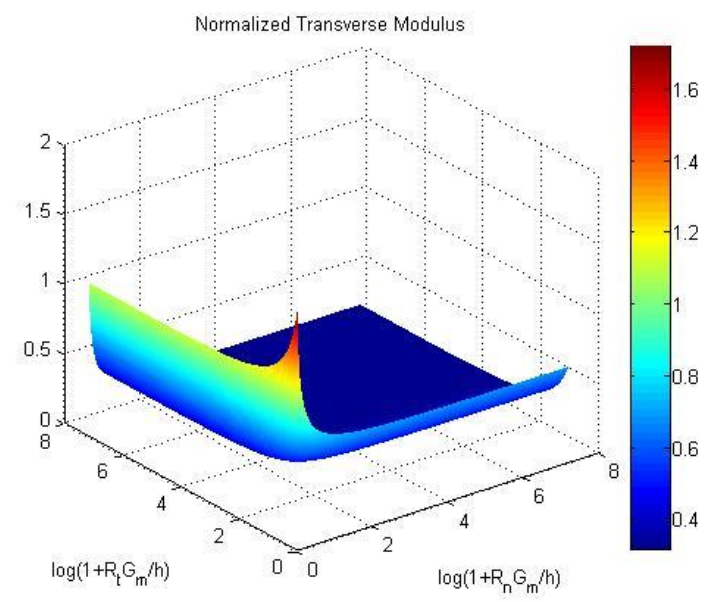

Figure 7.Transverse modulus of glass / epoxy composite with increasing tangential and normal separation parameters.

In order to apply the cell method to the properties determination in practical applications, it is necessary to determine the upper and lower $R_{t}, R_{n}$ because there is no accurate knowledge of the discontinuities at the common boundary after the scanning electron microscopy process. 
Theoretically, the $R_{t}$ tangential separation parameter theory has little effect on the normal elastic modulus and, on the other hand, has the most influence on the axial shear modulus $\left(G_{A}\right)$.The normal separation parameter $R_{n}$ has the greatest effect on transverse modulus and transverse shear modulus. The following range can be achieved by executing MATLAB code for different values of $R_{n}$ and $R_{t}$, observing the reduction properties:

$$
\begin{aligned}
& 0 \leq R_{t} \leq 1000 \\
& 0 \leq R_{n} \leq 1000
\end{aligned}
$$

Properties changes in values greater than $R_{t}, R_{n}>1000$ are less than $10^{-7}$.

Now, to investigate the impact of matrix micro-cracks, the hardness reduction is calculated in terms of the density of $D_{1}$ fibers perpendicular to the $D_{2}$ fibers parallel to the glass / epoxy system. According to Fig. 8, the transverse modulus volume increases with increasing volume and decreases with increasing modulus density. According to the figure it can be concluded that in the worst case the transverse modulus reduction is less than $1 \mathrm{GPa}$ because the transverse loads are parallel to the micro-crack surfaces. The greater impact of failure at higher volumetric percentages may be due to the interaction between the stress concentration of the fibers and the microtubules. As shown in Fig. 9, the longitudinal modulus decreases with increasing breakdown density at high volumetric percentages because as the composite reinforcement percentage increases, the fibers withstand higher hardness than the load matrix. Fig. 10 also shows the transverse modulus of the glass / epoxy composite in terms of volume ratio and different amounts of micro-crack density parallel to the fibers. As would be expected, the effect of micro-cracks parallel to the fibers on the transverse modulus is significant because the transverse load imposed on the composite is perpendicular to the micro-crack plates and causes it to open. It can also be deduced from Fig. 10 that the effect of parallel micro-crack densities with $D_{2}$ fibers is higher at high volumetric percentages, which may be due to the increase of stress concentration in the inter-matrix fibers. 


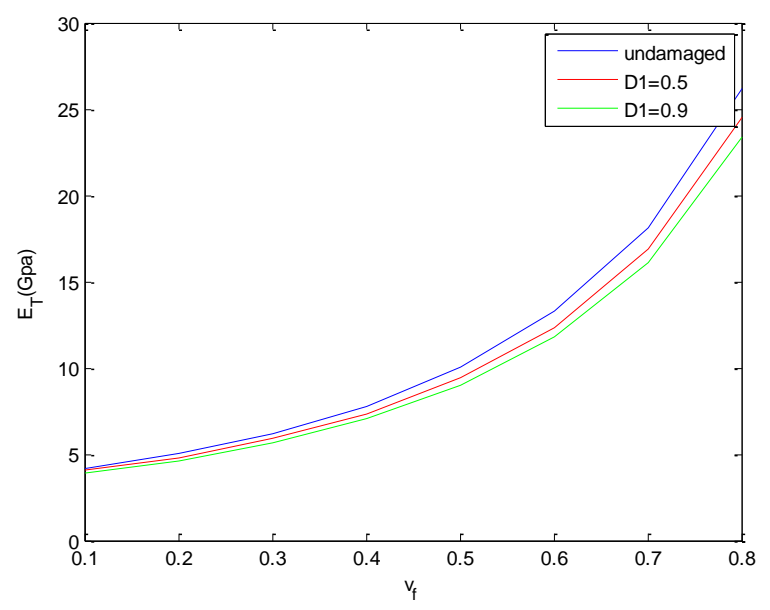

Fig. 8 Changes of transverse modulus of glass / epoxy composite in terms of volume percentage and density of perpendicular microcirculatory fibers

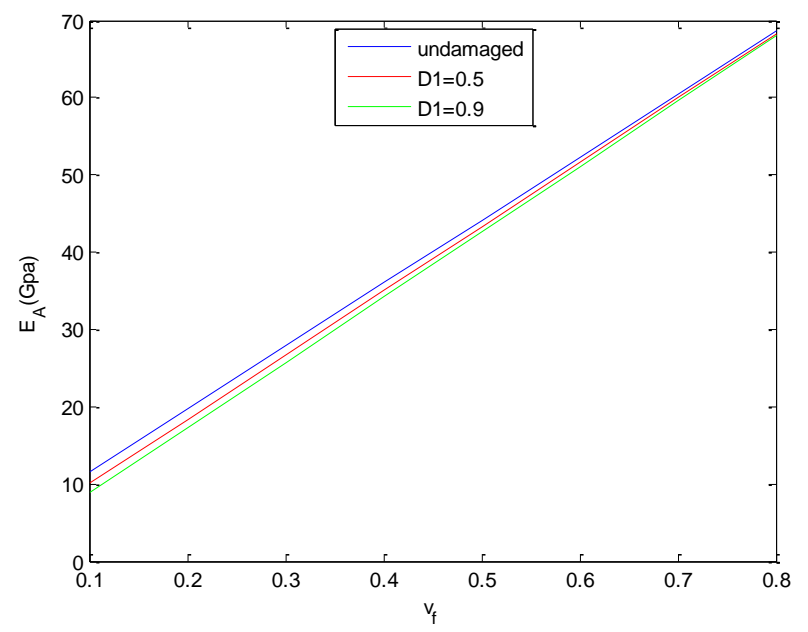

Fig. 9 Changes of longitudinal modulus of glass / epoxy composite in terms of volume and density of perpendicular microcirculatory fibers 


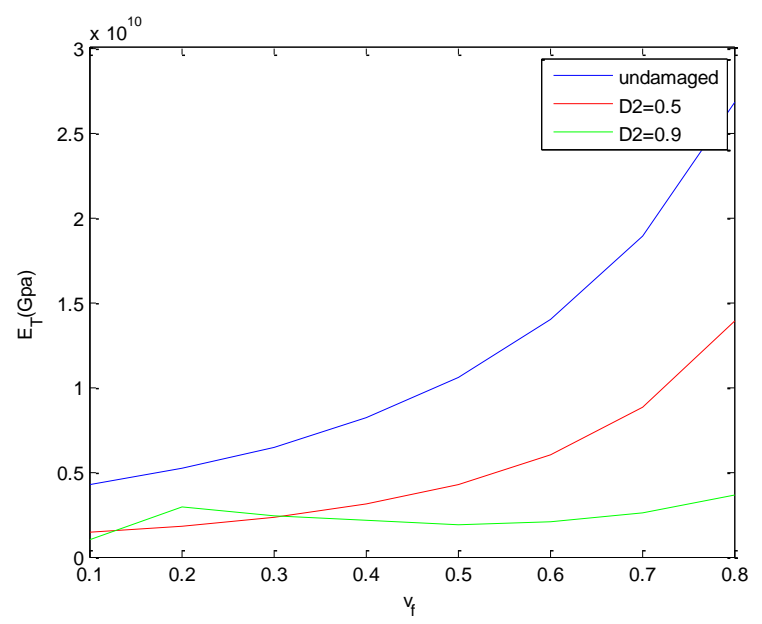

Fig. 10 Changes of transverse modulus of glass / epoxy composite in terms of volume percentage and density of perpendicular micro-crack fibers

\section{Conclusion}

In this study, the elastic properties of composite materials were determined using the cell method. In particular, the effect of incomplete connection between Leaf-Matrix common boundaries was introduced by introducing scalar parameters in the formulation of cell method. In the following, using the framework of mechanical failure of continuous environments, the effect of matrix micro-cracks on reducing the hardness of the composite was also discussed. The results of the properties change for the glass and boron / aluminum epoxy system show that the tangential and normal separation, respectively, significantly reduces the axial shear modulus and the transverse modulus of a composite. Changes in breakdown density were then investigated and it was observed that increasing the breakdown density reduced the transverse and longitudinal composition of the composite so that increasing the volumetric percentage of the fibers compensated for this decrease for the longitudinal module. Finally, the method of cells and the integration of scalar parameters in the expression of the common boundary separation phenomenon and matrix failure can be a good approximation for practical applications in determining the properties of composite multilayers with complex and perforated geometries. In other words, the elastic properties calculated at the single-layer surface can be entered as the input of a homogeneous material in the finite element software and used in multi-scaling analysis.

\section{References}

1. Callister, W.D.; Rethwisch, D.G. Materials science and engineering; John wiley \& sons NY, 2011; Vol. 5;. 
2. Reuß, A. Berechnung der fließgrenze von mischkristallen auf grund der plastizitätsbedingung für einkristalle. ZAMM-Journal Appl. Math. Mech. für Angew. Math. und Mech. 1929, 9, 49-58.

3. Voight, W. Theoretische Studien uber die Elasticitatsverhaltnisse des Krystalle, I, II. Abh. Ges. Wiss., Gottingen 1887.

4. Eshelby, J.D. The determination of the elastic field of an ellipsoidal inclusion, and related problems. Proc. R. Soc. London. Ser. A. Math. Phys. Sci. 1957, 241, 376-396.

5. Mori, T.; Tanaka, K. Average stress in matrix and average elastic energy of materials with misfitting inclusions. Acta Metall. 1973, 21, 571-574.

6. Hill, R. A self-consistent mechanics of composite materials. J. Mech. Phys. Solids 1965, 13, $213-222$.

7. Halpin, J.; Tsai, S. Environmental factors estimation in composite materials design, Air Force Materials Lab; 1967;

8. Fish, J. Multiscale methods: bridging the scales in science and engineering; Oxford University Press on Demand, 2010;

9. Kanouté, P.; Boso, D.P.; Chaboche, J.L.; Schrefler, B.A. Multiscale methods for composites: a review. Arch. Comput. Methods Eng. 2009, 16, 31-75.

10. Galvanetto, U.; Aliabadi, M.H. Multiscale modeling in solid mechanics: computational approaches; World Scientific, 2010; Vol. 3;.

11. Júnior, M.V.; de Souza Neto, E.A.; Munoz-Rojas, P.A. Advanced computational materials modeling: from classical to multi-scale techniques; John Wiley \& Sons, 2011;

12. Bednarcyk, B.; Aboudi, J.; Arnold, S. Micromechanics Modeling of Composite Materials Subjected to Multiaxial Progressive Continuum Damage. In Proceedings of the 50th AIAA/ASME/ASCE/AHS/ASC Structures, Structural Dynamics, and Materials Conference 17th AIAA/ASME/AHS Adaptive Structures Conference 11th AIAA No; 2009; p. 2547.

13. Jones, J.P.; Whittier, J.S. Waves at a flexibly bonded interface. 1967.

14. Kachanov, L.M. On creep rupture time. Izv. Acad. Nauk SSSR, Otd. Techn. Nauk 1958, 8, $26-31$.

15. Lemaitre, J. Chaboche, Jean-Louis. Mech. solid Mater. Edinburgh Build. Cambridge CB2 8RU, UK Cambridge Univ. Press 1990.

16. Aboudi, J. Micromechanical analysis of composites by the method of cells. 1989. 
17. Pickett, G. Elastic moduli of fiber reinforced plastic composites; Interscience New York, 1968; 